\title{
Hemopressin an Inverse Agonist of Cb1 Cannabinoid Receptors Reverses Mechanical Sensitivity on Diabetes-Induced Neuropathy in Mice
}

\author{
Elaine FlamiaToniolo ${ }^{1,2}$, Adriano CardozoFranciosi ${ }^{1,2}$ and Camila SquarzoniDale ${ }^{2 *}$ \\ ${ }^{1}$ DepartamentofPharmacology, Universityof Sao Paulo, Sao Paulo, Brazil; Hospital Sirio-Libanes, Brazil \\ ${ }^{2}$ Laboratory of Neuromodulation and Experimental Pain, Department of Anatomy, University of Sao Paulo, Brazil
}

\begin{abstract}
Peripheral neuropathy is one of the most common complications of diabetes affecting about $50 \%$ of patients with the disease. The most prominent symptoms involve the extremities and occur as both an exaggerated response to noxious stimuli (hyperalgesia), mild or non-painful stimuli (allodynia). Hemopressin ( $\mathrm{Hp}$ ) is a non apeptide first found in rat brain extracts, which selectively binds CB1 cannabinoid receptors (CB1R) and exerts antinociceptive actions in experimental inflammatory and neuropathic pain models. However there is no data about its efficacy in neuropathic metabolic-related disease, like diabetes mellitus. The aim of this study was to investigate the role of $\mathrm{Hp}$ on mechanical and thermal sensitivity of mice submitted to an experimental model of type 1 diabetes mellitus-induced neuropathy. Mechanical allodynia and thermal sensibility were assessed by von Frey filaments or plantar test, respectively, 7, 14, 21 and 28 days after streptozotocine injection (STZ; $200 \mathrm{mg} / \mathrm{kg}$ ). Body weight and blood glucose were monitored once a week. Hp was administered orally, once a day $(2.5 \mathrm{mg} / \mathrm{kg})$ for 28 days. Hp reversed mechanical allodynia in diabetic mice without changing blood glucose levels or body weight. No effects were observed for thermal sensitivity. These results make hemopressin an attractive approach for the development of cannabinoid-based therapies for the treatment diabetic neuropathic pain.
\end{abstract}

Keywords: Hemopressin; Diabetic neuropathy; Cannabinoid receptor; Mechanical allodynia; Thermal sensitivity; Mice

Abbreviations: Hp: Hemopressin; STZ: Streptozotocin; Sal: Saline; DPN: Diabetic Neuropathy

\section{Introduction}

Diabetic peripheral neuropathy (DPN) caused by diabetes mellitus is one of the most common complications of diabetes affecting about $50 \%$ of patients with the disease [1,2]. Among the many symptoms of this neuropathy, the development of chronic pain is one of the major complications on ceits emergence depends on multifactorial components, which are still poorly understood [3]. This neuropathic pain typically involves the extremities and is characterized by spontaneous and evoked pain stimuli with changes in pain perception, increased sensitivity to noxious stimuli (hyperalgesia) and sensitivity to light stimuli or stimuli that previously were not painful (allodynia). These factors strongly affect the quality of life of patients with this syndrome [4-7].

A precise cellular mechanism for the hyperalgesia and allodynia in the DPN is not yet known thus, the existing treatments, including anticonvulsant drugs or tricyclic antidepressant drugs [8-11], are still ineffective and unsatisfactory and only a few patients with DPN benefit from some pain relief $[1,4,12,13]$. An alternative therapy that has gained clinical acceptance is the use of compounds that modulate cannabinoid receptors, once these receptors are expressed in both neurons and microglia of rats with diabetic neuropathy, at both spinal and supraspinall evels [11,14-16].

The use of derivatives from Cannabis sativa $(\Delta 9-\mathrm{THC})$ for the treatment of various neurological disorders, including chronic pain, is supported by experimental and clinical data [17-20]. Although they are seen as promising target for the development of medications, clinical and preclinical studies have shown that $\triangle 9$-THC and other CB1 ligands generally produce undesirable effect in the Central Nervous System. CB1 agonists are generally at risk for psychoactive effects and dependence, limiting the optimization of doses in clinical trials and preclinical studies [21-23]. Thus, the development of drugs capable of binding to the cannabinoid receptors without psychoactive effects provides therapeutic potential without the risk of adverse effects [22].

Hemopressin (Hp), a nonapeptide (PVNFKFLSH) derived from the hemoglobin al chain was previously shown to target $\mathrm{CB}_{1}$ receptor, and to modulate its signaling [24-26]. Hp exhibits antinociceptive effect in inflammatory pain models [26,27]. Hp inhibits carrageen an-induced hyperalgesia only at the injured paw; without presenting antinociceptive effect in the contralateral, uninflamed paw, indicating that the effect of Hp is limited to tissue injury induced pain [26]. Also, intrathecal administration of $\mathrm{Hp}$ induces significant antinociception in the first and second phases of the formalin test [27]. The effects of $\mathrm{Hp}$ on carrageenan-induced hyperalgesia are independent of route of administration (oral, local, or intrathecal) [26]. More interesting is the fact that neurological side effects that are typically associated with antinociceptive doses of $\mathrm{CB}_{1}$ receptor ligands, including hypothermia, catalepsy and hypoactivity, were not reported with antinociceptive doses of Hp [26]. This, taken with the fact that the effects of Hp on carrageenan-induced hyperalgesia were found to be independent of route of administration, raises the possibility that $\mathrm{Hp}$ could be developed as a novel class of drug that modulates $\mathrm{CB}_{1}$ receptor for the treatment of pain. This study aimed to examine the effect of $\mathrm{Hp}$

*Corresponding author: Camila Squarzoni Dale, Department of Anatomy University of São Paulo, Av. Prof. Lineu Prestes, 2415, S104B, 05508-900, São Paulo, Brazil, Tel/Fax: + 55113091 0884, E-mail: camila.dale@usp.br

Received February 25, 2014; Accepted March 24, 2014; Published March 27, 2014

Citation: Toniolo EF, Franciosi AC, Dale CS (2014) Hemopressin an Inverse Agonist of $\mathrm{Cb} 1$ Cannabinoid Receptors Reverses Mechanical Sensitivity on Diabetes-Induced Neuropathy in Mice. J Diabetes Metab 5: 357 doi:10.4172/21556156.1000357

Copyright: ( $) 2014$ Toniolo EF, et al. This is an open-access article distributed under the terms of the Creative Commons Attribution License, which permits unrestricted use, distribution, and reproduction in any medium, provided the original author and source are credited. 
Citation: Toniolo EF, Franciosi AC, Dale CS (2014) Hemopressin an Inverse Agonist of Cb1 Cannabinoid Receptors Reverses Mechanical Sensitivity on Diabetes-Induced Neuropathy in Mice. J Diabetes Metab 5: 357 doi:10.4172/2155-6156.1000357

Page 2 of 5

treatment on mechanical and thermal sensitivity in a mouse model of diabetic neuropathy induced by streptozotocin (STZ).

\section{Materials and Methods}

\section{Animals}

Male C57BL6 mice weighing 20-25 g, age-matched, were used throughout this study. Animals were maintained under controlled light cycle $(12 / 12 \mathrm{~h})$ and temperature $\left(21 \pm 2^{\circ} \mathrm{C}\right)$ with free access to food and water. Throughout the experiments, animals were managed using the principles and guidelines for the care of laboratory animals in studies involving pain and were approved by the Ethics Committee on the Use of Animals at University of São Paulo (CEUA, protocol number 157/2011). Animals were divided in four groups: control saline group $(\mathrm{Sal}+\mathrm{Sal})$, streptozotocin group $(\mathrm{STZ}+\mathrm{Sal}), \mathrm{Hp}$ group $(\mathrm{Sal}+\mathrm{Hp})$ and $\mathrm{STZ} / \mathrm{Hp}$ group (STZ+Hp)

\section{Diabetic Neuropathy(DPN)}

Mice received a single intraperitoneal injection of streptozotocine (STZ; 200 mg/Kg, body weight; Sigma, St. Louis, MO) dissolved in sterile $0.9 \%$ saline after 4 hours of food restriction. Control animals received only sterile $0.9 \%$ saline injection. The fasting blood glucose levels were measured, after a fasting period of $4 \mathrm{~h}$, by glucometer (ACCU-CHEK, Roche Diagnostics)before STZ or Sal injection and again on days 7, 14, 21 and 28 after STZ. Mice whose blood glucose levels exceeded $300 \mathrm{mg} /$ dl were considered diabetic [28].

\section{Behavioral analysis}

Mechanical allodynia - von Frey test: Testing for mechanical allodynia (Von Frey filaments - Touch-Test Sensory Evaluators North Coast Medical) was performed according to the method of Chaplan [29]. Mice were placed individually in plastic cages with a wire bottom, which allowed access to their paws. To reduce stress, mice were habituated to the experimental environment one day before the first measurement. At the day of the test, the animals were placed in

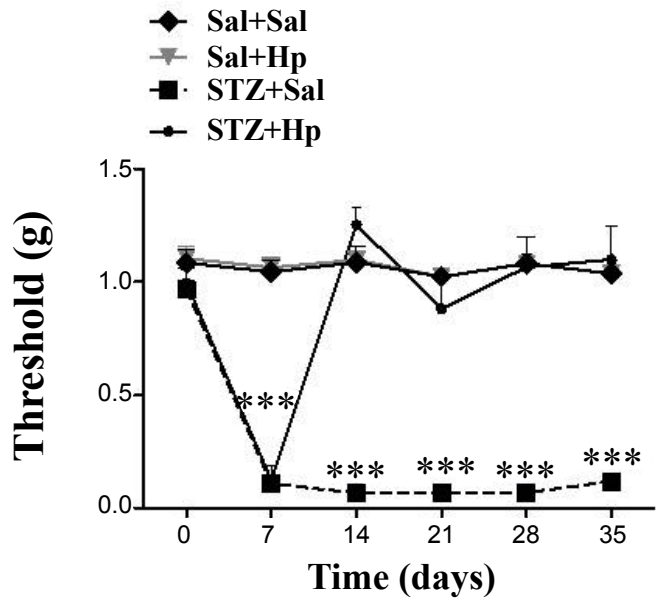

Figure 1: Effect of Hp on mechanical sensitivity of diabetic mice evaluated by von Frey filaments.

Animals were evaluated before any treatment (time 0 ), after 7 days of streptozotocin (STZ) or saline (Sal) injection, and after treatment with hemopressin $(\mathrm{Hp})$ or Sal. HP was administered once a day, orally at a dose of $2.5 \mathrm{mg} / \mathrm{kg}$ for 28 days. Data are mean \pm S.E.M. Ganimals per group. ${ }^{* * *} p$ $<0.001$ vs Sal+Sal and Sal+Hp group. (Two-way ANOVA followed by Bonferron post-test). the cages 30 min before the beginning of each measurement. The area tested was the mid-plantar left paw.

Thermal sensitivity - Plantar test: Assessment of sensitivity to the thermal stimulus was based on the method of Hargreaves et al. [30] with instrumentation provided by IITC Life Science. Animals were placed for 30 minutes in compartments of $15 \mathrm{~cm}^{2}$ surface with a transparent glass for the habituation process. To reduce stress, mice were habituated to the experimental environment one day before the first measurement. The glass allows the passage of the light beam, which can lead to painful thermal stimuli, adjusted to $30 \%$ of the maximum intensity of heating. Test was conducted after a pre-habituation thermal sensitivity baseline. The beam was directed to the plantar surface of the left hind paw (the thermal stimulus was applied for a maximum period of $20 \mathrm{sec}$ to prevent tissue damage in the animal's paw). Latency in seconds to withdraw the paw was considered as nociceptive response. Three measurements were made for each hindpaw, with an interval of 5 minutes between them. The mean of the measurements was used as the thermal nociceptive response.

\section{Hemopressin}

Hemopressin (Proteimax Biotechnology) was administered orally at the dose of $2.5 \mathrm{mg} / \mathrm{kg}$ [26,31], body weight diluted insterile $0.9 \%$ saline solution for 28 days. Control animals received only $0.9 \%$ saline solution injection.

\section{Statistics}

Results are presented as the mean \pm standard error of the mean (SEM). Statistical analyses of data were generated using GraphPad Prism, version 4.02 (GraphPad Software Inc., San Diego, CA, USA). Statistical comparison of more than two groups was performed using analysis of variance (ANOVA), followed by Bonferroni's test. Statistical comparison for treatment over time was performed using two way ANOVA followed by Bonferroni's test. In all cases, $\mathrm{p} \leq 0.05$ was considered statistically significant.

\section{Results}

\section{Hp inhibits mechanical sensitivity on diabetic mice}

STZ induced mechanical allodyniain mice from the $7^{\text {th }}$ day after STZ injection up to 28 days $(\mathrm{n}=6)$ (Figure 1). Once confirmed the presence of neuropathic pain, at the $7^{\text {th }}$ day groups of animals $(n=6)$ received oral treatment with $\mathrm{Hp}$ for 28 days and presented a full reversion of mechanical sensitivity in all evaluated days, which was not observed in the group of mice treated with saline $(n=6)$ (Figure 1). Furthermore, a control saline group that was also treated with Hp showed no changes on pain sensitivity when compared to the initial measurement or with the Saline+Saline group ( $\mathrm{n}=6$ ) (Figure1).

\section{Treatment with Hp does not interfere with thermal sensitivity of diabetic mice}

Mice treated with STZ $(n=5)$ showed a loss on pain sensitivity (hypoalgesia)to the thermal stimulation in all evaluated times when compared with Sal+ Sal group $(n=5)$ (Figure 2). Also, treatment with Hp was not able to reverse STZ-induced thermal hypoalgesia $(n=5)$ (Figure 2).

Thermal sensitivity of mice was measured before the injection of STZ or Saline (time 0), 7 days after STZ or Sal and 28 days after treatment with $\mathrm{Hp}$ or Sal. 
Citation: Toniolo EF, Franciosi AC, Dale CS (2014) Hemopressin an Inverse Agonist of Cb1 Cannabinoid Receptors Reverses Mechanical Sensitivity on Diabetes-Induced Neuropathy in Mice. J Diabetes Metab 5: 357 doi:10.4172/2155-6156.1000357

Page 3 of 5

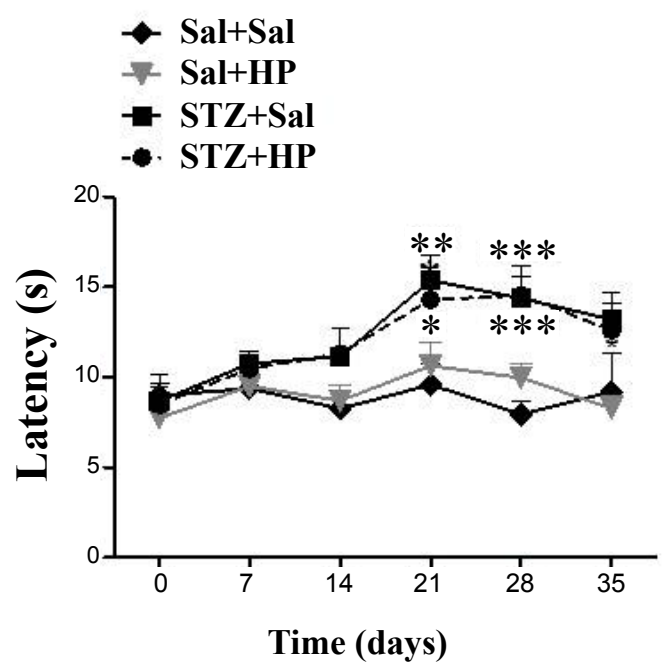

Figure 2: Effect of $\mathrm{Hp}$ on thermal sensitivity of diabetic mice evaluated by the plantar test.

Mice were evaluated before any treatment (time 0 ), after 7 days of streptozotocin (STZ) or saline (Sal) injection, and after treatment with hemopressin $(\mathrm{Hp})$ or Sal. HP was administered once a day, orally at a dose of $2.5 \mathrm{mg} / \mathrm{kg}$ for 28 days. Data are mean \pm S.E.M. 5animals per group. ${ }^{*} p<0.05,{ }^{* *} p<0.01$ and ${ }^{* * *} p$ $<0.001$ compared to group Sal+Sal and Sal+Hp. (Two-way ANOVA followed by Bonferroni post-test)

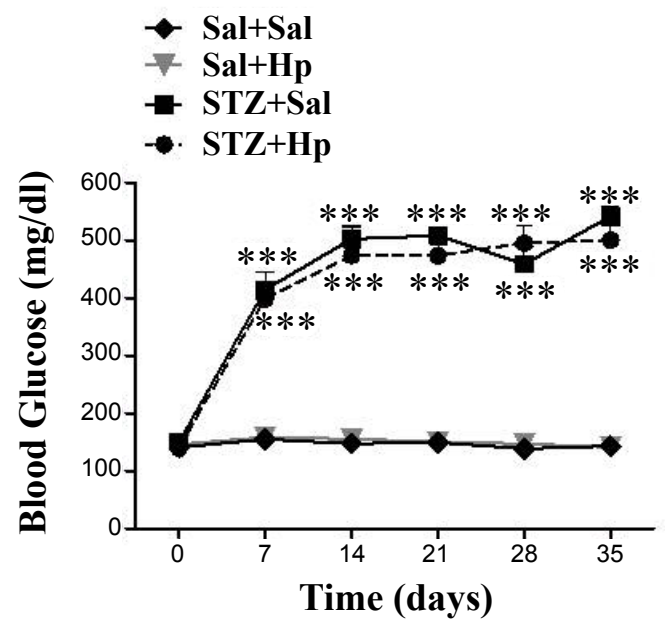

Figure 3: Effect of Hp on blood glucose levels of diabetic mice.

Glucose levels were measured prior to any treatment (time 0), 7 days after STZ or Sal injection and after $7,14,21$ and 28 days after $\mathrm{Hp}$ treatment. Data are mean \pm S.E.M. 11 animals per group. ${ }^{* * *} \mathrm{p}<0.001$ vsSal+SalorSal+Hp groups. (Two-way ANOVA followed by Bonferroni post-test).

\section{Effect of HP treatment on blood glucose levels of diabetic mice}

Serum glucose levels were checked before any treatment (time 0 ), 7 days after STZ or Salinjection to verify the presence of hyperglycemia or normal glucose levels, and after 7, 14, 21 and 28 days of Hp treatments.

STZ $(n=11)$ was effective in inducing diabetes on mice observed by the increase on blood glucose levels of animals when compared with Sal-treated mice $(n=11)$. Hp treatment did not interfere with the increase on blood glucose levels of STZ mice $(n=11)$. Furthermore, Hp by itself didn't cause any changes in control animals $(n=11)$ (Figure 3$)$.

\section{Monitoring of changes on body weight of diabetic mice treated with $\mathrm{Hp}$}

During the experiments, animalswere weighed before any treatment (time 0), 7 days after STZ or Sal injection and after 7, 14, 21 and 28 days after HP or Sal treatment. STZ-injected mice $(n=11)$ presented a decrease on body weight that was not modified by Hp-treatment $(n=11)$ (Figure 4). Also, Hp did not compromise the weight gain in the control group (n=11) (Figure 4).

\section{Discussion}

In this study, it was evaluated the antinociceptive effect of $\mathrm{Hp}, \mathrm{a} \mathrm{CB}_{1}$ receptor ligand, $\mathrm{Hp}$ in a mice model of diabetic-induced neuropathy [28]. Results presented herein demonstrate that STZ-induced diabetic mice showed a decrease on mechanical pain threshold when compared to non-diabetic (saline) mice, in all evaluated times. This result is consistent with previous reports from literature demonstrating that diabetic mice show a low pain threshold, thus characterizing the diabetic neuropathy as a model of neuropathic pain [21]. We found that 28 days of oral treatment withHpcould completely block signs of mechanical pain from the $7^{\text {th }}$ day after $\mathrm{Hp}$ administration, supporting the idea that Hp induces true antinociception in this neuropathic pain model. These data corroborate with data obtained by our group demonstrating that oral treatement with Hp completely blocks signs of pain (hyperalgesia and allodynia) in rats with neuropathic pain induced by the chronic constriction of the sciatic nerve [31]. In addition, the effect observed herein was maintained for $24 \mathrm{~h}$ after suspension of $\mathrm{Hp}$, suggesting that a longer treatment with $\mathrm{Hp}$, maintain a more lasting effect on the prevention of pain [31]. These results are consistent with data published in previous reports using carrageenan-induced hyperalgesia test and the writhing test where different doses of $\mathrm{Hp}$ were found to produce the same inhibitory effect on pain sensitivity [32] and that administration of $\mathrm{Hp}$ by oral or intraperitoneal or intraplantar route achieve similar level of analgesia [26] supporting its oral bioavailability. A possible explanation for this phenomenon is the tendency to aggregate in the Hp [33] which could protect the peptide from rapid degradation in an in vivo "environment" and thus maintain the analgesic effect observed.

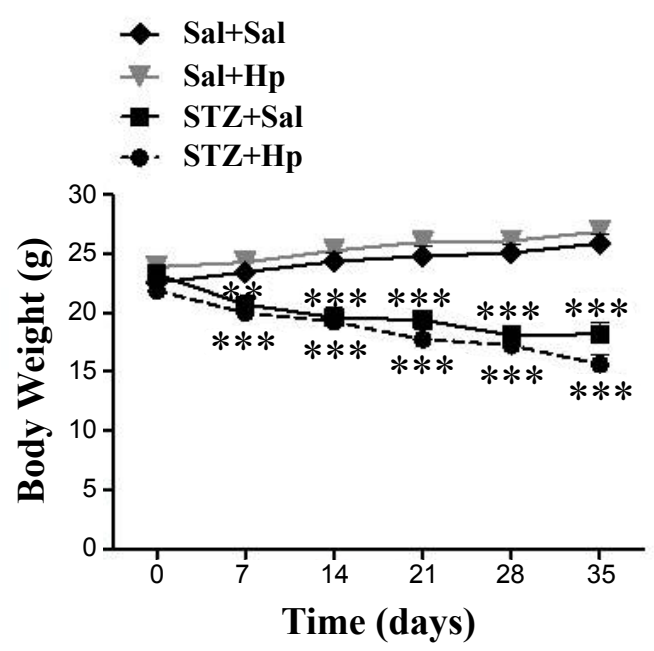

Figure 4: Monitoring of body weight of diabetic mice treated with $\mathrm{Hp}$ Body weight was monitored prior to any treatment (time 0), 7 days after STZ or Sal injection and after $7,14,21$ or 28 days of HP treatment. Data are mean \pm S.E.M. 11 animals per group. ${ }^{* *} p<0.01,{ }^{* * *} p<0.001$ (Two-way ANOVA followed by Bonferroni post-test) 
Conflicting data have been reported concerning the thermal nociceptive threshold in STZ-induced diabetes model. While some studies reported thermal hyperalgesia, others observed thermal hypoalgesia or normal thermal thresholds after STZ injections. This may be in part due to methodological details such as use of different species, limbs, and methods of heat application. In this sense it is demonstrated that insulin-deficient diabetic rats with the plantar surface of the hind paw exposed to a temperature ramp rising from $30^{\circ} \mathrm{C}$ at a rate of $1^{\circ} \mathrm{C} / \mathrm{s}$ over $20 \mathrm{~s}$, exhibit a transient thermal hyperalgesia during the first few weeks of diabetes. It progresses to thermal hypoalgesia within 2-3 months [34]. This is also common in mice submitted to the STZ model. Some mice injected with streptozotocin $(50-200 \mathrm{mg} / \mathrm{kg})$ does not become hyperglycemic but exhibit a transient thermal hyperalgesia when evaluated in the hot plate test, that returned to normal within weeks. This contrasts with hyperglycemic mice that progressed from hyperalgesia to hypoalgesia [13,28]. In our present study, mice displayed thermal hypoalgesia 1 week after injection of streptozotocin and subsequent induction of diabetes. This phenomena was mantained up to 5 weeks of observation. This result is similar to data from human diabetic patients where there is a progression from painful to degenerative painless neuropathy, although at present it is not clear that thermal hypoalgesia in diabetic rats or mice coincides with any loss of epidermal thermal nociceptors, as appears to be the case in diabetic patients [35]. In this work we demosntrate that Hp treatment was not able to interfere with thermal hypoalgesia. However, Hp treatment didn't modify thermal sensitivity on control mice injected with saline, thus reforcing that $\mathrm{Hp}$ is specific in treating mechanical hyperalgesia observed on diabetes.

Another interesting finding was the fact that although treatment with $\mathrm{Hp}$ has been effective in reversing the painful picture, no changes were observed regarding the blood glucose levels or body weight of animals, which continued to progress with the development of diabetes. Moreover, Hp treatment by itself did not cause any change in the control group of animals, demonstrating that $\mathrm{Hp}$ is specific on treating painful neuropathy.

Recent data demonstrated that $\mathrm{Hp}$ treatement interfere with the transmission of neuropathic pain message to the central nervous system, reducing nociceptor activation in spinal cord [31]. Also, Hp inhibits calcium mobilization in dorsal root ganglia neurons from both normal and neuropathic rats, reinforcing the idea that Hp modulates primary afferent nociceptive signal by inhibiting sensory neurons [31]. Recent data showed that hemopressin activates distinct neuronal substrates within the brain, focused mainly on the feeding-related circuits of the mediobasal hypothalamus and in nociceptive regions of the periaqueductal grey (PAG) and dorsal raphe (DR). In contrast to AM251, there is a distinct lack of activation of the brain reward centres, such as the ventral tegmental area, nucleus accumbens and orbitofrontal cortex, which normally form a functional activity signature for the central action of synthetic CB1 receptor inverse agonists. Thus, hemopressin modulates the function of key feeding-related brain nuclei of the mediobasal hypothalamus, and descending pain pathways of the PAG and DR, and not higher limbic structures. Thus, hemopressin may offer behaviourally selective effects on nociception and appetite, without engaging reward pathways [36]. These data complement the existing data in the literature showing that that neurological responses that are typically associated with antinociceptive doses of CB1R ligands, including hypothermia, catalepsy and hypoactivity, were not reportedwithantinociceptive doses of $\mathrm{Hp}$ [26]. Another interesting point is that the effects observed for Hp depend on its aminoacid sequence, once it was demonstrated that central administration of an extended form of Hp, VD-PVNFKFLSH (VD-Hp) induces dosedependent antinociception in mice but also induces undesired effects on the Central Nervous System such as tolerance to antinociception and conditioned place aversion [37]. Taken together, these data raises the possibility that Hp could be developed as a novel class of drug that modulates CB1R for the treatment of pain.

In conclusion, our data demonstrates that $\mathrm{Hp}$ exhibits antinociceptive properties on diabetes-induced peripheral neuropathy. This effect is specific for the treatment of chronic neuropathic pain. Although the mechanisms involved in the effects of hemopressin need to be further characterized, the results obtained so far suggest a role for a cannabinoid-like compound in regulating chronic neuropathic pain that could be further explored to develop therapeutic drugs based on the hemopressin sequence.

\section{Acknowledgement}

This work was supported by Hospital Sírio-Libanês, FAPESP grant (2012/50176-5 to CSD) andCoordenação de Aperfeiçoamento de Pessoal de Nível Superior (CAPES to EFT). The authors declare no conflicts of interest.

\section{References}

1. Boulton AJ, Vinik Al, Arezzo JC, Bril V, Feldman EL, et al. (2005) Diabetic neuropathies: a statement by the American Diabetes Association. Diabetes Care 28: 956-962

2. Feldman EL, Stevens MJ, Russell JW (2005) The Diabetes Mellitus Manual 6: 366-384.

3. Cheng HT, Dauch JR, Oh SS, Hayes JM, Hong Y, et al. (2010) p38 mediates mechanical allodynia in a mouse model of type 2 diabetes. Mol Pain 6: 28.

4. Barrett AM, Lucero MA, Le T, Robinson RL, Dworkin RH, et al. (2007) Epidemiology, public health burden, and treatment of diabetic periphera neuropathic pain: a review. Pain Med 8 Suppl 2: S50-62.

5. Cao XH1, Byun HS, Chen SR, Cai YQ, Pan HL (2010) Reduction in voltagegated $\mathrm{K}+$ channel activity in primary sensory neurons in painful diabetic neuropathy: role of brain-derived neurotrophic factor. J Neurochem 114: 14601475.

6. Rondón LJ1, Privat AM, Daulhac L, Davin N, Mazur A, et al. (2010) Magnesium attenuates chronic hypersensitivity and spinal cord NMDA receptor phosphorylation in a rat model of diabetic neuropathic pain. J Physiol 588 4205-4215.

7. Said G (2007) Diabetic neuropathy--a review. Nat Clin Pract Neurol 3: 331-340.

8. Attal N (2000) Chronic neuropathic pain: mechanisms and treatment. Clin J Pain 16: S118-130.

9. Choucair-Jaafar N, Salvat E, Freund-Mercier MJ, Barrot M4 (2014) The antiallodynic action of nortriptyline and terbutaline is mediated by $\hat{\imath}^{2}(2)$ adrenoceptors and $\hat{l}^{\prime}$ opioid receptors in the ob/ob model of diabetic polyneuropathy. Brain Res 1546: 18-26.

10. Galer BS (1995) Neuropathic pain of peripheral origin: advances in pharmacologic treatment. Neurology 45: S17-25.

11. Sah DW1, Ossipo MH, Porreca F (2003) Neurotrophic factors as nove therapeutics for neuropathic pain. Nat Rev Drug Discov 2: 460-472.

12. Tesfaye S, Kempler P (2005) Painful diabetic neuropathy. Diabetologia 48: 805 807.

13. Vinik Al (1999) Diabetic neuropathy: pathogenesis and therapy. Am J Med 107: 17S-26S.

14. Aley KO, Levine JD (2002) Different peripheral mechanisms mediate enhanced nociception in metabolic/toxic and traumatic painful peripheral neuropathies in the rat. Neuroscience 111: 389-397.

15. Erichsen HK, Blackburn-Munro G (2002) Pharmacological characterisation of the spared nerve injury model of neuropathic pain. Pain 98: 151-161.

16. Toth CC1, Jedrzejewski NM, Ellis CL, Frey WH 2nd (2010) Cannabinoidmediated modulation of neuropathic pain and microglial accumulation in a model of murine type I diabetic peripheral neuropathic pain. Mol Pain 6: 16. 
Citation: Toniolo EF, Franciosi AC, Dale CS (2014) Hemopressin an Inverse Agonist of Cb1 Cannabinoid Receptors Reverses Mechanical Sensitivity on Diabetes-Induced Neuropathy in Mice. J Diabetes Metab 5: 357 doi:10.4172/2155-6156.1000357

Page 5 of 5

17. Boyd ST (2006) The endocannabinoid system. Pharmacotherapy 26: 218S-221S

18. Di Marzo V, Petrosino S (2007) Endocannabinoids and the regulation of their levels in health and disease. Curr Opin Lipidol 18: 129-140.

19. Iversen L1, Chapman V (2002) Cannabinoids: a real prospect for pain relief? Curr Opin Pharmacol 2: 50-55.

20. Millan MJ (1994) Serotonin and pain: evidence that activation of 5-HT1A receptors does not elicit antinociception against noxious thermal, mechanical and chemical stimuli in mice. Pain 58: 45-61.

21. Lichtman AH1, Martin BR (1991) Cannabinoid-induced antinociception is mediated by a spinal alpha 2-noradrenergic mechanism. Brain Res 559: 309314

22. McCarberg BH1, Barkin RL (2007) The future of cannabinoids as analgesic agents: a pharmacologic, pharmacokinetic, and pharmacodynamic overview. Am J Ther 14: 475-483.

23. Takahashi KA1, Castillo PE (2006) The CB1 cannabinoid receptor mediates glutamatergic synaptic suppression in the hippocampus. Neuroscience 139 795-802.

24. Dale CS, Pagano Rde L, Rioli V (2005) Hemopressin: a novel bioactive peptide derived from the alpha1-chain of hemoglobin. Mem Inst Oswaldo Cruz 100 Suppl 1: 105-106.

25. Gomes I1, Grushko JS, Golebiewska U, Hoogendoorn S, Gupta A, et al. (2009) Novel endogenous peptide agonists of cannabinoid receptors. FASEB J 23: 3020-3029.

26. Heimann AS1, Gomes I, Dale CS, Pagano RL, Gupta A, et al. (2007) Hemopressin is an inverse agonist of CB1 cannabinoid receptors. Proc Natl Acad Sci U S A 104: 20588-20593.

27. Hama A1, Sagen J (2011) Centrally mediated antinociceptive effects of cannabinoid receptor ligands in rat models of nociception. Pharmacol Biochem Behav 100: 340-346.
28. Davidson E, Coppey L, Lu B, Arballo V, Calcutt NA, et al. (2009) The Roles of Streptozotocin Neurotoxicity and Neutral Endopeptidase in Murine Experimental Diabetic Neuropathy. Experimental Diabetes Research 43: 1980.

29. Chaplan SR1, Bach FW, Pogrel JW, Chung JM, Yaksh TL (1994) Quantitative assessment of tactile allodynia in the rat paw. J Neurosci Methods 53: 55-63.

30. Hargreaves K1, Dubner R, Brown F, Flores C, Joris J (1988) A new and sensitive method for measuring thermal nociception in cutaneous hyperalgesia. Pain 32: 77-88.

31. Toniolo EF, Maique ET, Ferreira-Jr WA, Heimann AS, Ferro ES, et al. (2014) Hemopressin inhibits neuropathic pain in rats. Peptides.

32. Dale CS, Pagano Rde L, Rioli V, Hyslop S, Giorgi R, et al. (2005) Antinociceptive action of hemopressin in experimental hyperalgesia. Peptides 26: 431-436.

33. Gomes I, Dale CS, Casten K, Geigner MA, Gozzo FC, et al. (2010) Hemoglobinderived peptides as novel type of bioactive signaling molecules. AAPS $\mathrm{J} 12$ 658-669.

34. Calcutt NA (2004) Experimental models of painful diabetic neuropathy. J Neurol Sci 220: 137-139.

35. Kennedy WR1, Wendelschafer-Crabb G, Johnson T (1996) Quantitation of epidermal nerves in diabetic neuropathy. Neurology 47: 1042-1048.

36. Dodd GT, Worth AA, Hodkinson DJ, Srivastava RK, Lutz B, et al. (2013) Central functional response to the novel peptide cannabinoid, hemopressin Neuropharmacology 71: 27-36.

37. Han ZL, Fang Q, Wang ZL, Li XH, Li N, et al. (2014) Antinociceptive effects of central administration of the endogenous cannabinoid receptor type 1 agonist VDPVNFKLLSH-OH [(m)VD-hemopressin(a)], an N-terminally extended hemopressin peptide.J Pharmacol Exp Ther348:316-323. 